Review

\title{
Purinergic mechanism in the immune system: A signal of danger for dendritic cells
}

\author{
Francesco Di Virgilio \\ Department of Experimental and Diagnostic Medicine, Section of General Pathology, and Interdisciplinary Center for \\ the Study of Inflammation (ICSI), University of Ferrara, Ferrara, Italy
}

Received 14 October 2004; accepted in revised form 3 January 2005

Key words: danger signals, dendritic cells, extracellular ATP, inflammation

\begin{abstract}
There is increasing appreciation that injured or stressed cells release molecules endowed with the ability to modulate dendritic cell maturation. The role of these molecules is thought to be that of alerting the body of an impending danger, and initiate and shape the subsequent immune response. Nucleotides are perfectly suited for this task as they are easily released upon damage of the cell membrane, rapidly diffuse in the extracellular environment and ligate specific plasma membrane receptors expressed by dendritic cells and other mononuclear phagocytes. A better knowledge of the modulation of dendritic cell responses by extracellular nucleotides may provide novel routes to enhance the immune response and increase the efficacy of vaccination.
\end{abstract}

Abbreviations: ssRNA - single strand RNA; TLR - toll-like receptors

\section{Introduction}

One of the fundamental tenets in immunology is that our body reacts against foreign (non-self) antigens. Antigenic molecules, that signal invasion by pathogenic microrganisms, or simply the presence of cells that carry a foreign genome (as in transplants) are initally sensed by first line defense immune cells (such as the dendritic cells, DCs, the prototypic antigen presenting cells, APCs) that initiate inflammation and the native and adaptive immune responses [1]. Albeit generally accepted and largely validated by countless experimental and clinical findings, the theory that we exclusively or mainly react to non-self molecules is unable to explain a number of observations that have bothered immunologists for many years. As pointed out by Matzinger [2], why we do not react against proteins that are synthesized late in the life and therefore are not exposed to lymphocytes during maturation of the immune system? Or, why do we all harbour auto-reactive B and T cells, and yet autoimmune diseases are rare? Or, why are some tissues easier to transplant than others? Over the last years, the view has emerged that we do not react to nonself ('foreign') molecules but rather to molecules that cause damage to our tissues. In other words, our body reacts to

Correspondence to: Dr Francesco Di Virgilio, Department of Experimental and Diagnostic Medicine, Section of General Pathology, and Interdisciplinary Center for the Study of Inflammation (ICSI), University of Ferrara, Ferrara, Italy. E-mail: FDV@UNIFE.IT 'danger' rather than to 'stranger' [3]. While it is clear that the 'danger hypothesis' does not rule out but rather complements non-self recognition in immunity, it is also clear that this novel way to look at the very first phases of the immune response gives some powerful weapons for the interpretation of many key events in immunity and inflammation.

Every physician and every pathologist knows well that the strongest stimulus for inflammation is tissue damage, to such an extent that cell injury is thought to be the common event whereby conditions as different as cancer, chemical irritants, UV light, closed traumas or biological agents trigger inflammation [4]. The pro-inflammatory ('flogistic') activity of tissue trauma was long thought to reside in the release of normally segregated intracellular molecules that once exteriorized into the extracellular environment would have the ability to switch on quiescent immune cells. Not surprisingly, it is well known that necrotic cells are more likely than apoptotic cells to initiate inflammation [5]. However, the identity of these intracellular molecules has been ill defined for many years, and the pathways leading to stimulation of native and adaptive immunity were purely hypothetical. The merit of the 'danger signal' hypothesis as put forward by Matzinger lays in the description of the basic features of a danger signal and in the identification of their role in the initiation of inflammation and in the modulation of DCs [6]. We will see that extracellular nucleotides have all the essential features of a 'danger signal', and in fact they may be a type of primordial signal of tissue distress ubiquitous in the biological world. 
Accordingly, nucleotide receptors (P2R) in the immune system can be viewed as receptors for danger signals.

\section{Danger signals: What are they?}

According to the current generally accepted definition, we may define a danger signal as something that is released by injured or dying cells, is perceived as a sign of tissue distress, and is endowed with the ability to stimulate DCs and induce their differentiation [6]. Danger signals can be endogenous (originating from the host's cells) or exogenous (released into the body by invading pathogens). Danger signals can also be generated by tissue destruction or activation of classical inflammatory pathways [7, 8]. Endogenous danger signals can further be classified into constitutive or inducible. A key feature of the constitutive danger signals is their ability to stimulate the release of the inducible ones. Constitutive danger signals are normally segregated inside all cells and, so far as they are intracellular, they have no stimulatory activity on the immune system. Several intracellular constituents are candidates to this role (see Table 1), among which are extracellular nucleotides.

A danger signal must be in the first place a good extracellular messenger because, in case of tissue damage, it must efficiently travel across the intercellular space to alert nearby cells. Ideally, any good extracellular messenger should have the following features:

1. It should be easily and quickly generated in the extracellular space. This would require the presence of a large pool of intracellular molecules.

2. Under resting conditions its concentration should be close to zero to allow a high signal-to-noise ratio upon release. Maintenance of a large pool and a low steadystate value of messenger molecules is an essential feature of signalling in biological systems because it allows a rapid, many-fold change in messenger concentration.

3. It should be highly mobile in the pericellular aqueous environment, therefore small and hydrophilic molecules are better suited to this task.

4. It should be recognized by specific receptors expressed by immune cells.

5. It should be easy to destroy once it reaches the extracellular space. This ensures a quick termination of the response once the causative agent is removed.

Table 1. Intracellular molecules candidates for the role of constitutive danger signals.

\begin{tabular}{ll}
\hline Ligand (danger signal) & Receptor (danger sensor) \\
\hline $\begin{array}{l}\text { Heat shock proteins } \\
\quad \text { HSP60, HSP70, HSP90) }\end{array}$ & CD91, TLR4, TLR4/TLR2, LOX-1 \\
Uric acid crystals & $?$ \\
DNA & TLR9 \\
ssRNA & TLR3, TLR7, TLR8 \\
Nucleotides & P2Y $1,2,4,6,11$, P2X \\
Sugar metabolites \\
(UDPglucose)
\end{tabular}

Extracellular nucleotides fulfill all these pre-requisites as:

1. They are present at a high concentration intracellularly (ATP 5-10 mM, UTP 0.5-1 mM), and are quickly released following cell death, damage, or even simple plasma membrane stretching. The mechanism by which ATP can be released in the absence of overt cell damage is unclear: it is well documented that some cell types (e.g. platelets [9], neurons [10] or epithelia [11]) secrete ATP most likely by exocytosis of nucleotide-laden vesicles. Evidence mostly based on inhibitor studies suggests that channels or transporters might also participate in ATP release (see [12] for recent review). More controversial is the evidence that pro-inflammatory factors may to drive non-lytic ATP release from immune cells [13-16].

2. The extracellular ATP concentration under resting conditions is very low, in the $1-10 \mathrm{nM}$ range. Paradoxically, before the purinergic signalling hypothesis became accepted, this was taken as evidence against the role of ATP as an extracellular messenger. On the contrary, a very low resting level is a fundamental feature of any messenger, danger signals included.

3. ATP is a small solute (molecular mass $650 \mathrm{Da}$ ) bearing from two to four negative charges, depending on the $\mathrm{pH}$ and the $\mathrm{Ca}^{2+}$ and $\mathrm{Mg}^{2+}$ concentration. Thus, it is highly diffusible in the extracellular aqueous milieu.

4. As of now 15 receptors for extracellular nucleotides have been identified [17]. The widely differing range of nucleotide specificity and affinity makes this receptor family very flexible and efficient in translating information delivered by the ligands.

5. All cells express plasma membrane ecto-nucleotidases that quickly hydrolyze extracellular nucleoside tri-, diand mono-phosphates [18]. Interestingly, these enzymes by generating nucleoside diphosphates from triphosphates (e.g. ADP from ATP, or UDP from UTP) and thus additional ligands also modulate activation of P2 receptors with different specificities.

\section{Are extracellular nucleotides released at sites of tissue damage?}

This may sound as a very naive question. We all believe that an acutely injured cell will release all its ATP content, as can be clearly shown in the test tube when a cell suspension (or a cell monolayer in a Petri dish) is lysed with a detergent or by freeze-thawing, and the ATP concentration in the supernatant is measured by the usual luciferin-luciferase assay. However, in vivo measurements that document an increase in the extracellular ATP concentration at sites of inflammation or tissue damage are few. An early in vivo report by Forrester [19] suggested that cell membrane stretching can cause ATP secretion. This author showed that the ATP concentration in the venous effluent from human exercising muscle increased from 20 to about $200 \mathrm{pmol} / \mathrm{ml}$, mainly due to release from 
contracting muscle fibers. Other measurements following vessel injury show that the free ATP concentration in the blood can rise up to $20 \mu \mathrm{M}$ from a basal level of $10-20 \mathrm{nM}$ [20]. The origin of this ATP is identified as the damaged vessel wall cells (endothelium and muscle cells), platelets and other circulating blood cells. Platelets are without doubt an important source of ATP since they store near molar amounts of this nucleotide inside their dense granules. It is worth pointing out that, although nucleotide release from platelets is generally viewed in the context of clot formation, we should not forget that platelets are also one of the most important sources of inflammatory mediators, thus ATP release from activated platelets is certainly proof for ATP release during inflammation. Implications for a typical inflammatory lesion in which platelets have a key role, such as the atheromatous plaque, could be far reaching [21].

A widely held opinion is that the main source of ATP release in the setting of trauma is the damaged cells. While this is very likely to occur, a recent study by Nedergaard and co-workers has documented that shortly $(10 \mathrm{~min})$ after the trauma the main site of ATP release is not the traumatized region itself, but rather the perilesional area [22]. It appears that cells that surround the traumatized tissue are driven into an activated state that persists for several hours after the injury. Quite interestingly, blockade of the $\mathrm{P} 2 \mathrm{X}_{7}$ receptor subtype, a main mediator of the proinflammatory effects of ATP, decreased tissue injury and enhanced functional recovery after the insult. This finding lends further support to the view that ATP might be one of the most important early pro-inflammatory signals released at injury sites. The view that local accumulation of ATP (and ADP) might have a long lasting action is further supported by the observation that in mice lacking the main Langerhans cell ecto-ATPase (CD39) local irritants cause nucleotide release and an exacerbated skin inflammation [23]. Furthermore, local accumulation of nucleotides also affects the outcome of contact dermatitis caused by topical administrations of allergens. This again concurs to support the view that nucleotide release at site of injury or inflammation can have far reaching effects on immunomodulation.

\section{Do nucleotides activate DCs?}

As summarized above, the main distinguishing feature of a danger signal resides in its ability to activate DCs and drive their differentiation. DCs are the most efficient APCs for activation of naive $\mathrm{T}$ cells [24]. Thus, they are the key elements in the initiation of primary immune response. DCs reside in the tissues as immature cells, but when activated by a variety of extracellular agents (pathogens, cytokines, neurotransmitters), they mature and migrate to the lymphonodes where they stimulate $\mathrm{T}$ cell differentiation $[1$, 24]. DCs have become a focus of attention as a possible target for extracellular nucleotides only very recently, nonetheless a wealth of data is already available on the pattern of P2R expression and the functional responses associated with their stimulation.
DCs express both P2YRs ( $\mathrm{P} 2 \mathrm{Y}_{1}, \mathrm{P} 2 \mathrm{Y}_{2}, \mathrm{P}_{2} \mathrm{Y}_{4}, \mathrm{P} 2 \mathrm{Y}_{6}$, $\mathrm{P} 2 \mathrm{Y}_{11}$ and $\left.\mathrm{P} 2 \mathrm{Y}_{14}\right)$ and $\mathrm{P} 2 \mathrm{XRs}\left(\mathrm{P} 2 \mathrm{X}_{1}, \mathrm{P} 2 \mathrm{X}_{4}, \mathrm{P} 2 \mathrm{X}_{7}\right)$ [25-30]. Acute, transient, stimulation with ATP or UTP causes many of the responses typically associated with P2Y or P2X activation (phosphatidyl inositol breakdown, release of $\mathrm{Ca}^{2+}$ from intracellular stores, $\mathrm{Ca}^{2+}$ influx across the plasma membrane, and even opening of the $\mathrm{P} 2 \mathrm{X}_{7}$ large conductance pore if a high ATP concentration is used).

Nucleotide effects on DC responses are dramatically different whether they are due to exposure to chronic low $(10-250 \mu \mathrm{M})$ or acute high $(500 \mu \mathrm{M}$ to millimolar) concentrations. Chronic stimulation triggers a process of DC maturation that enhances their ability to stimulate Th2 lymphocytes and thus initiate a type 2 immune response. In the presence of ATP the expression of typical markers (CD54, CD80, CD83, CD86) of DC maturation is increased, furthermore endocytic activity is reduced and the capacity to promote proliferation of allogeneic lymphocytes enhanced. ATP alone, at variance with classical maturation factors, does not induce cytokine or chemokine secretion $[31,32]$. However, in the presence of maturating factors (e.g. bacterial endotoxin, LPS, or CD40L), ATP strongly distorts the pattern of cytokine produced. LPS or CD40L-dependent release of IL-1 $\beta$, IL-1 $\alpha$, TNF- $\alpha$, IL-6 and IL-12 is inhibited, while release of the IL-1 receptor antagonist and IL-10 is not affected. As a result, when cocultured with naïve Th lymphocytes these DCs promote a Th2 polarization, witnessed by the typical pattern of cytokines produced by the stimulated lymphocytes (low interferon $\gamma$, high IL-4, IL-5 and IL-10). The pattern of chemokine receptors expressed by DCs is also strongly modified by chronic exposure to low ATP concentrations. Receptors for inflammatory chemokines such as CC chemokine receptor 5 (CCR5) are down modulated while those for lymphoid chemokines such as CXC chemokine receptor 4 (CXC4R) and CCR7 are upregulated [33]. This has far reaching consequences in recirculation of dendritic cells from tissues to the lymphonodes, and from blood into tissues. In fact, following exposure to pathogens or tissue damage, resident immature DCs respond to inflammatory chemokines to become activated, concentrate in the inflamed tissue, take up antigens, mature and migrate to the regional lymphonodes. During maturation DCs upmodulate receptors for lymphoid chemokines, that allow lymphonode localization and enhance their ability to interact with naïve $T$ cells. ATP priming also affects the pattern of chemokines secreted by DCs: constitutive production of the lymphoid chemokine CCL22 is increased, while the LPS-stimulated secretion of the inflammatory chemokines CXCL10 and CCL5 is inhibited [33]. This shift in chemokine release favours recruitment of Th2 rather than Th1 lymphocytes. Thus, in summary chronic exposure to low ATP concentrations has a strong modulatory activity on DC maturation by enhancing their ability to localize in the lymphonodes and to initiate a type 2 rather than a type 1 immune response (see Figure 1 for a schematic rendering). The P2Rs responsible for such an immunomodulatory effect have not been conclusively identified, but convergent data point to $\mathrm{P} 2 \mathrm{Y}_{11}$ as the most likely candidate [29] 


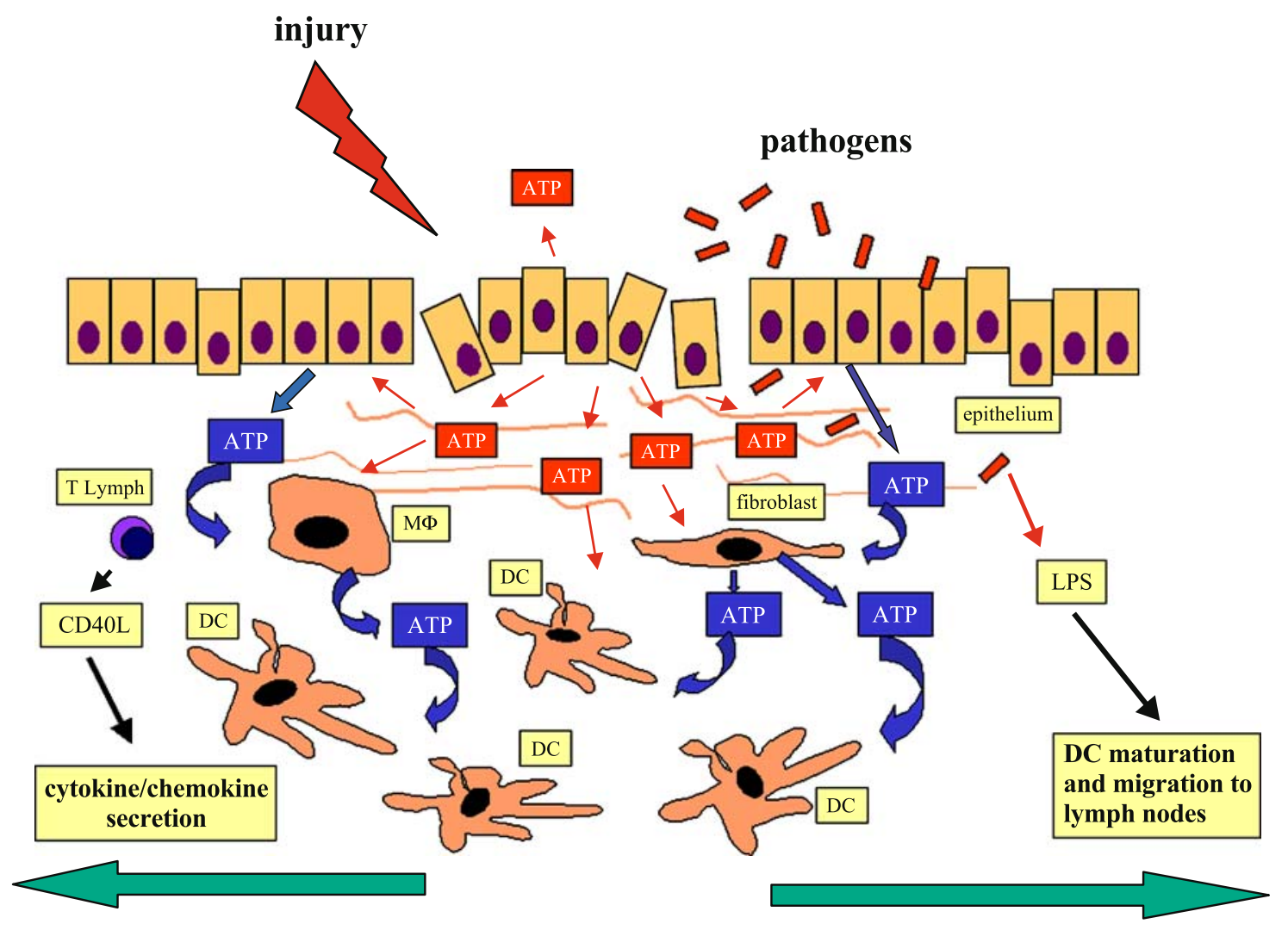

Figure 1. Cell damage caused by physical trauma or pathogens causes an initial wave of ATP release (red) from injured cells. This first wave of ATP release diffuses through the pericellular space and activates adjacent cells (e.g. epithelial cells, macrophages, fibroblasts). P2 receptors expressed on the plasma membrane of the bystander cells will be activated and trigger the release of several bioactive agents, among which ATP itself (ATP-induced ATP release, dark blue). Accumulation of ATP into the extracellular milieu will prime Langerhans/dendritic cells (DC) and modify their responses to bacterial endotoxin (LPS) or T lymphocyte-derived CD40 ligand (CD40L). The combined action of these agents drives DC maturation and the secretion of cytokines and chemokines.

(despite an early suggestion that P2XRs were involved [31]). However, at this very preliminary stage we cannot exclude that other P2YRs or P2XRs may also participate in DC maturation. Involvement of different P2Rs in DC maturation is also suggested by a recent work of Schnurr and co-workers [34] showing that ATP-primed DCs have a differential ability to activate naive or memory $\mathrm{T}$ cells. This further stresses the complexity and the potential relevance of purinergic signalling in the manipulation of DC responses.

We are faced with a completely different scenario when DCs are acutely challenged with high ATP doses in association with a bacterial pathogen. In this case secretion of pro-inflammatory cytokines is potently stimulated and, if exposure to ATP is not discontinued, cell death occurs $[27,35]$. This pro-inflammatory activity is non dissimilar from that elicited by ATP in many other mononuclear phagocytes [36, 37]. The IL-1 $\beta$-releasing activity of ATP is particularly striking: in cells primed with LPS the addition of this nucleotide causes an explosive acceleration of proIL-1 $\beta$ processing and the consequent exteriorization of the mature cytokine embedded into plasma membrane-derived microvesicles [38]. It is of interest that ATP is unable to drive IL-1 $\beta$ release in phagocytes that have not been previously primed with LPS, thus reinforcing ATP's role as danger signal that acts in concert with bacterial pathogens to initiate inflammation. On the other hand, the inability of LPS to drive IL- $1 \beta$ secretion in the absence of ATP further supports the view that bacterial factors are incomplete stimuli in the absence of endogenously-released danger signals.

Nucleotides may shape the immune response also by having a differential effect on DC recruitment. Idzko and co-workers have shown that immature but not mature DCs chemotact in response to a nucleotide gradient [39]. This would allow recruitment of immature DCs to the ATP-rich inflammatory site, but would not prevent migration of mature DCs to regional lymph nodes. Many other immune cells, such as mast cells, macrophages and microglia, are sensitive to the chemotactic activity of ATP, UTP or ADP, thus reinforcing the role of nucleotides as powerful activators of inflammation [40-42]. As of now there are no reports of an in vivo modulatory activity of extracellular ATP on dendritic cell function, but the observations reported in mice deleted of the main ecto-ATPase of immune cells (CD39) point in this direction [23].

\section{Conclusion}

The danger model for the initiation of the immune response is enjoying increasing appreciation. Although overenthusi- 
astic generalizations should be avoided, we think that it provides a useful guidance to comprehension of the initial phases of inflammation. In this context, nucleotides are an outstanding example of how an intracellular molecule, with no obvious links to immunity, can modulate the immune response if released extracellularly.

\section{Acknowledgements}

This work was supported by grants from the Ministry of Education and Scientific Research (Cofin, FIRB), the Italian Association for Cancer Research (AIRC), and institutional funds from the University of Ferrara.

\section{References}

1. Bancherau J, Steinman RM. Dendritic cells and the control of immunity. Nature 1998; 3(92): 245-52.

2. Matzinger P. Tolerance, danger and the extended family. Annu Rev Immunol 1994; 12: 991-1045.

3. Heath WR, Carbone FR. Dangerous liaisons. Nature 2003; 425: 460-1.

4. Kumar V, Cotran RS, Robbins SL. Basic pathology. Philadelphia: Saunders 2002.

5. Gallucci S, Lolkema M, Matzinger P. Natural adjuvants: Endogenous activators of dendritic cells. Nat Med 1999; 5: 1249-55.

6. Gallucci S, Matzinger P. Danger signals: SOS to the immune system. Curr Opin Immunol 2001; 13: 114-9.

7. Aliberti J, Viola JP, Vieira-de-Abreu A et al. Bradykinin induces IL12 production by dendritic cells: Danger signal that drives $\mathrm{TH} 1$ polarization. J Immunol 2003; 170: 5349-53.

8. Skoberne M, Beignon A-S, Bhardwaj N. Danger signals: A time and space continuum. Trends Mol Med 2004; 10: 251-7.

9. Fukami MH, Salganicoff L. Human platelet storage organelles. A review. Thromb Haemost 1977; 38: 963-70.

10. Burnstock G. Noradrenaline and ATP as cotransmitters in sympathetic nerves. Neurochem Int 1990; 17: 357-68.

11. Homolya L, Steinberg TH, Boucher RC. Cell to cell communication in response to mechanical stress via bilateral release of ATP and UTP in polarized epithelia. J Cell Biol 2000; 150: 1349-60.

12. Schwiebert EM, Zsembery A. Extracellular ATP as a signalling molecule for epithelial cells. Biochim Biophys Acta 2003; 1615: 7-32.

13. Bodin P, Burnstock G. Increased release of ATP from endothelial cells during acute inflammation. Inflamm Res 1998; 47: 351-4.

14. Ferrari D, Chiozzi P, Falzoni $\mathrm{S}$ et al. Purinergic modulation of interleukin-1 beta release from microglial cells stimulated with bacterial endotoxin. J Exp Med 1997; 185: 579-82.

15. Bal-Price A, Moneer Z, Brown GC. Nitric oxide induces rapid, calcium-dependent release of vesicular glutamate and ATP from cultured rat astrocytes. Glia 2002; 40: 312-323

16. Beigi RD, Dubyak GR. Endotoxin activation of macrophages does not induce ATP release and autocrine stimulation of P2 nucleotide receptors. J Immunol 2000; 165: 7189-98.

17. Di Virgilio F, Baricordi OR, Romagnoli R, Baraldi PG. Leukocyte P2 receptors: A novel target for anti-inflammatory and anti-tumor therapy. Curr Drug Targets 2004; 5: 85-99.

18. Zimmermann H. Extracellular metabolism of ATP and other nucleotides. Naunyn-Schmiedebergs Arch Pharmacol 2000; 362: 299-309.

19. Forrester T. An estimate of adenosine triphosphate release into the venous effluent from exercising human forearm muscle. J Physiol 1972; 224: 611-28.

20. Borne GVR, Kratzer MAA. Source and concentration of extracellular adenosine triphospate during hemostasis in rats, rabbits and man. J Physiol 1984; 354: 419-29.
21. Di Virgilio F, Solini A. P2 receptors: New potential players in atherosclerosis. Br J Pharmacol 2002; 135: 831-42.

22. Wang X, Arcuino G, Takano T et al. P2X7 receptor inhibition improves recovery after spinal cord injury. Nature Med 2004; 10: 821-7.

23. Mizumoto N, Kumamoto T, Robson SC et al. CD39 is the dominat Langerhans cell-associated ecto-NTPdase: Modulatory roles in inflammation and immune responsiveness. Nature Med 2002; 8: 358-65.

24. Cella M, Sallusto F, Lanzavecchia A. Origin, maturation and antigen presenting function of dendritic cells. Curr Opin Immunol 1997; 9: 10-6.

25. Mutini C, Falzoni S, Ferrari D et al. Mouse dendritic cells express the $\mathrm{P}_{2} \mathrm{X}_{7}$ purinergic receptor: Characterization and possible participation in antigen presentation. J Immunol 1999; 163: 1958-65.

26. Berchtold S, Ogilvie AL, Bogdan $\mathrm{C}$ et al. Human monocyte derived dendritic cells express functional P2X and P2Y receptors as well as ecto-nucleotidases. FEBS Lett 1999; 458: 424-428.

27. Ferrari D, la Sala A, Chiozzi P et al. The P2 purinergic receptors of human dendritic cells: Identification and coupling to cytokine release. FASEB J 2000; 14: 2466-76.

28. Schnurr M, Then F, Galambos P, et al. Extracellular ATP and TNF- $\alpha$ synergize in the activation and maturation of human dendritic cells. J Immunol 2001; 165: 4704-9.

29. Wilkin F, Duhant X, Bruyns C et al. The P2Y11 receptor mediates the ATP-induced maturation of human monocyte-derived dendritic cells. J Immunol 2001; 166: 7172-7.

30. Skelton L, Cooper M, Murphy M, Platt A. Human immature monocyte-derived dendritic cells express the $\mathrm{G}$ protein-coupled receptor GPR105 (KIAA0001, $\mathrm{P}_{2} \mathrm{Y}_{14}$ ) and increase intracellular calcium in response to its agonist, uridin diphosphoglucose. J Immunol 2003; 171: 1941-9.

31. La Sala A, Ferrari D, Corinti S et al. Extracellular ATP induces a distorted maturation of dendritic cells and inhibits their capacity to initiate Th1 responses. J Immunol 2001; 166: 1611-7.

32. Wilkin F, Stordeur P, Goldman M et al. Extracellular adenine nucleotides modulate cytokine production by human monocytederived dendritic cells: Dual effect on IL-12 and stimulation of IL10. Eur J Immunol 2002; 32: 2409-17

33. La Sala A, Sebastiani S, Ferrari D et al. Dendritic cells exposed to extracellular adenosine triphosphate acquire the migratory properties of mature cells and show a reduced capacity to attract type 1 lymphocytes. Blood 2002; 99: 1715-22.

34. Schnurr M, Toy T, Shin A et al. Extracellular nucleotide signalling via P2 receptors inhibits IL-12 and enhances IL-23 expression in human dendritic cells: A novel role for the cAMP pathway. Blood 2005; 105: 2465-72.

35. Coutinho-Silva R, Persechini P, Bisaggio RD et al. $\mathrm{P} 2 \mathrm{Z} / \mathrm{P} 2 \mathrm{X}_{7}$ receptor-dependent apoptosis of dendritic cells. Am J Physiol 1999; 276: C1139-47.

36. Perregaux D, Gabel CA. Interleukin-1 beta maturation and release in response to ATP and nigericin. Evidence that potassium depletion mediated by these agents is a necessary and common feature of their activity. J Biol Chem 1994; 269: 15195-203.

37. Ferrari D, Villalba M, Chiozzi P, Falzoni S, Ricciardi-Castagnoli P, Di Virgilio F. Mouse microglial cells express a plasma membrane pore gated by extracellular ATP. J Immunol 1996; 156: 1531-9.

38. MacKenzie A, Wilson HL, Kiss-Toth E et al. Rapid secretion of interleukin-1beta by microvesicle shedding. Immunity 2001; 15: 825-35.

39. Idzko M, Dichmann S, Ferrari D et al. Nucleotides induce chemotaxis and actin polymerization in immature but not mature human dendritic cells via activation of pertussis toxin-sensitive P2y receptors. Blood 2002; 100: 925-32.

40. McCloskey MA, Fan Y, Luther S. Chemotaxis of rat mast cells toward adenine nucleotides. J Immunol 1999; 163: 970-7.

41. Oshimi Y, Miyazaki S, Oda S. ATP-induced $\mathrm{Ca}^{2}+$ response mediated by P2U and P2Y purinoceptors in human macrophages: Signalling from dying cells to macrophages. Immunology 1999; 98: 220-7.

42. Honda S, Sasaki Y, Ohsawa K et al. Extracellular ATP or ADP induce chemotaxis of cultured microglia through Gi/o-coupled P2Y receptors. J Neurosci 2001; 21: 1975-82. 